\title{
Rationale for blood request: cross match versus group and screen
}

\author{
Chanika A Gamage', P Pratheepan ${ }^{2}$, S Sivaganesh ${ }^{1,2}$ \\ ${ }^{1}$ Department of Surgery, Faculty of Medicine, University of Colombo \\ ${ }^{2}$ Professorial Surgical Unit, National Hospital of Sri Lanka
}

Key words: Blood products; crossmatch; group and save

\begin{abstract}
Introduction

Adherence to guidelines for the cross-matching and reservation of blood for surgical procedures will optimize the utilization of resources. The objective of this study was to evaluate the practice of cross-match and utilization of blood for elective surgeries in a tertiary Sri Lankan surgical unit using the following criteria: cross-match to transfusion ratio $(\mathrm{C} / \mathrm{T}$ ratio $) \leq$ 3.0, transfusion probability $(\% \mathrm{~T}) \geq 30$ and transfusion index $(\mathrm{TI}) \geq 0.5$.
\end{abstract}

\section{Material and methods}

A retrospective analysis of 104 patients who underwent elective surgical procedures at the Professorial Surgical Unit, Colombo during the period of January-September 2011 was done. Data was obtained from patient records. Blood utilization was evaluated using the following indices: $\mathrm{C} / \mathrm{T}$ ratio, $\% \mathrm{~T}$ and TI.

\section{Results}

Of the 104 patients, $96(92.3 \%)$ had a cross-match and $8(7.69 \%)$ had only a group and screen. The cumulative non-utilisation of cross-matched blood was 107/126 (84.9\%).All procedures studied had a $\mathrm{CT}$ ratio $>3$ and a $\mathrm{TI}<0.5$ i.e. beyond the criteria of justification for a crossmatch. Large bowel surgeries, mastectomies and oesophagectomies marginally matched the criteria justifying a crossmatch only in respect to the $\% \mathrm{~T}$, with values just above $30 \%$.

Correspondence : Gamage CA

Email : chanika_ga2005@yahoo.com

\section{Conclusion}

This data suggests that unwarranted cross-matching of blood is done in most procedures, especially cholecystectomies, fundoplications and thyroidectomies where a group and screen is adequate. We recommend further study of this issue and the development of evidence based blood ordering schedules in each hospital.

\section{Introduction}

Blood products are resources vital to healthcare institutions with constant and growing demands. The ability to maintain adequate stocks of blood products to meet its needs can be a challenge to any major hospital, especially when rare groups are involved. Perioperative transfusion requirements for surgical procedures, both elective and emergency, constitute a significant component of utilised blood products. However, anecdotal and published evidence seems to indicate that requests for blood products in elective surgical settings are excessive and that only a small proportion of cross matched blood or its derivatives is ultimately utilized [1-3]. This imposes an avoidable strain on national and local blood transfusion services both in terms of workload, utilisation of resources and the need to replenish stocks with donors.

The reservation of blood products for elective surgical procedures is carried out using two procedures, i.e. by a group and save only or a routine crossmatch. Performing a group and save involves the patient's blood sample being grouped $(\mathrm{A}, \mathrm{B}, \mathrm{O}$ or $\mathrm{AB})$ and screened for common antibodies (other than anti-A or anti-B) responsible for donor red cell haemolysis. Performing a routine cross match involves doing a group and save and checking for compatibility with blood packs reserved for transfusion by directly 
mixing donor red cells with recipient plasma. A routine group and save with a crossmatch takes up to two hours to perform and the requested packs are reserved for the specific patient for 72 hours. Inappropriate or excessive crossmatching therefore results in the blood being unavailable for the use of other patients for the specified time period, ensuing in inventory issues for blood banks, loss of shelf life and potential wastage of blood available in emergencies. In addition, the extra time and reagents utilized for crossmatching increases the financial burden on already stretched healthcare budgets.

The tendency to over request blood for elective surgical procedures is often guided by habit and 'hospital routines' rather than actual clinical need or evidence based criteria. Subsequent non-utilization and wastage is defended by the excuse that it is a safety measure in case of an excessive or unexpected blood loss during surgery $[3,6]$. Adherence to guidelines for the crossmatching and reservation of blood for elective surgical procedures will optimize the utilization of resources.

The introduction of guidelines for the reservation of blood for specific elective surgical procedures such as the maximum surgical blood ordering schedule (MSBOS)have been shown to minimize the problem of inappropriate crossmatching and over requesting of blood $[2,4,5,7]$. They enable identification of procedures that can be carried out with a group and save only instead of a crossmatch and reservation of blood. ${ }^{4,8}$ Studies have concluded that an individual MSBOS should be established at each hospital by a hospital transfusion committee which represents both blood providers and users after evaluating their hospital's own transfusion practices $[1,4]$. Standardized indices such as a crossmatch to transfusion ratio $(\mathrm{C} / \mathrm{T}$ ratio $) \leq 3.0$, transfusion probability $(\% \mathrm{~T}) \geq 30$ and transfusion index $(\mathrm{TI}) \geq 0.5$ have been used to justify procedure specific crossmatching of blood and the establishment of institutional MSBOS $[2-4,9,10]$.

Despite a number of studies done elsewhere, to the authors' knowledge there are no published accounts of local practice in this area. The aim of this study was to evaluate the practice of crossmatch and utilization of blood for elective surgical procedures in a Sri Lankan tertiary surgical unit and make recommendations based on the findings.

\section{Materials and methods}

A retrospective analysis was carried out among 104 patients who underwent elective surgical procedures in a tertiary surgical unit at the National Hospital of Sri Lanka Colombo, during the time period of JanuarySeptember 2011. Patients who had received a preoperative blood transfusion were excluded from the study. Data on patient demography, number of units cross matched or grouped and saved, number of units transfused and the surgical procedure performed were obtained from patient records available at the central record room.

The utilization of blood was calculated using the following standardized indices [4].

- Crossmatch to transfusion ratio $(\mathrm{CT}$ ratio $)=$ number of units cross matched/ number of units transfused

- $\quad$ Transfusion probability $(\% \mathrm{~T})=$ (number of patients transfused / number of patients crossmatched) $\mathrm{x} 100$

- Transfusion Index (TI) = number of units transfused / number of patients cross matched

\section{Results}

Out of the 104 patients there were 34 males and 70 females with their age ranging from 15 to 85 years. Of the 104 patients who underwent elective surgical procedures, 96 patients $(92.3 \%)$ had a crossmatch while 8 patients $(7.7 \%)$ had only a group and save. A total number of 126 units were cross matched for 96 patients. Only 14 of the 96 patients were transfused a total of 19 units of blood, the cumulative nonutilization of crossmatched blood being 107/126 (84.9\%).

Table 1 summarises the procedure specific number of patients and the units of blood crossmatched and transfused for each group.

The procedure specific utilization of blood was calculated using the standardized indices $\mathrm{CT}$ ratio, $\% \mathrm{~T}$ and TI as indicated in the materials and methods (Table 2).

\section{Discussion}

Blood and its derivatives play a major role in the management of surgical patients. Overestimation of anticipated blood loss during surgery leads to over 
reservation of blood, resulting in the wastage of valuable resources [4]. The historical practice of a routine crossmatch for major elective surgical procedures was likely borne out of real transfusion requirements in that era. However, at present, a better understanding of the physiological basis of optimal tissue perfusion and advances in methods of surgical haemostasis have led to a reduction in both perioperative blood loss and transfusion requirements. Despite this, the practice of routine crossmatching in unwarranted cases appears to continue probably driven by habit and a perceived sense of safety in the event of unexpected haemorrhage $[3,6]$. Moreover, routine reservation of blood is done by the most junior member of the surgical team, sometimes at the behest of a junior anaesthetist, both of whose appreciation of the potential for significant haemorrhage for a particular procedure may be limited [5].

Inappropriate reservation of blood results in its reduced availability for other patients in need and a greater tendency for it to pass its expiry date and be discarded [5]. Morphological and biochemical changes that occur during storage have adverse effects on erythrocyte viability and function following transfusion leading to a limited shelf life of 42 days for red cell units [2]. At the same time, crossmatching is time consuming and expensive [3].Therefore, the practice of blood reservation and transfusion in surgical practice should be subject to regular evaluation in healthcare institutions.

The crossmatch to transfusion (CT) ratio, the transfusion probability $(\% \mathrm{~T})$ and the transfusion index (TI) are indices employed to evaluate utilization of blood for surgical procedures $[1,9,10]$. A crossmatch is justified in procedures that carry a $\mathrm{CT}$ ratio $<3, \mathrm{a} \% \mathrm{~T}$ $>30 \%$ or a $\mathrm{TI}>0.5[2-4,9,10]$.

This study revealed that for the given period in this unit the overall $\mathrm{CT}$ ratio was $6.63, \% \mathrm{~T}$ was $14.58 \%$ and $\mathrm{TI}$ was 0.197 , all of which fall outside the accepted criteria that justify a crossmatch. In effect, there was non-utilization or in effect over reservation of 107 units of red cells $(84.92 \%)$.

Further analysis also showed that certain procedures such as laparoscopic or open fundoplication, laparoscopic or open cholecystectomy and total or hemithyroidectomy did not require any blood transfusion despite significant amounts of blood being

\begin{tabular}{|l|c|c|c|c|}
\hline \multicolumn{1}{|c|}{ Procedure } & $\begin{array}{c}\text { Patients cross } \\
\text { matched }\end{array}$ & $\begin{array}{c}\text { Units cross } \\
\text { matched }\end{array}$ & $\begin{array}{c}\text { Patients } \\
\text { transfused }\end{array}$ & $\begin{array}{c}\text { Units } \\
\text { transfused }\end{array}$ \\
\hline Laparoscopic / open fundoplication & 3 & 4 & 0 & 0 \\
\hline Laparoscopic / open cholecystectomy & 21 & 22 & 0 & 0 \\
\hline Total / hemithyroidectomy & 26 & 27 & 0 & 0 \\
\hline Matectomy \& acillary clearence & 10 & 12 & 3 & 3 \\
\hline Oesophagectomy & 3 & 8 & 1 & 1 \\
\hline Large bowel surgery & 33 & 53 & 10 & 15 \\
\hline Cumulative & 96 & 126 & 14 & 19 \\
\hline
\end{tabular}

Table 1-Procedure specific cross match and transfusion data

\begin{tabular}{|l|c|c|c|}
\hline \multicolumn{1}{|c|}{ Procedure } & CT Ratio & $\% \mathrm{~T}$ & TI \\
\hline Laparoscopic / open fundoplication & $4: 0$ & 0.00 & 0.00 \\
\hline Laparoscopic / open cholecystectomy & $22: 0$ & 0.00 & 0.00 \\
\hline Total / hemithyroidectomy & $27: 0$ & 0.00 & 0.00 \\
\hline Mastectomy \& axillary clearance & $12: 3(4)$ & 30.00 & 0.30 \\
\hline Oesophagectomy & $8: 1(8)$ & 33.30 & 0.33 \\
\hline Large bowel surgery & $53: 15(3.5)$ & 30.30 & 0.45 \\
\hline Cumulative & $126: 19(6.6)$ & 14.60 & 0.20 \\
\hline
\end{tabular}

Table 2 -Procedure specific blood utilization indices 
reserved.

The criteria justifying blood reservation were met for only large bowel surgery (including anterior resection, abdomino-perineal resection and hemicolectomy), mastectomy and oesophagectomy. Even for the above three procedures, transfusion was only marginally justifiable with respect to transfusion probability $(\% \mathrm{~T})$, with values for the crossmatch to transfusion (CT) ratio and the transfusion index (TI)falling beyond the accepted levels.

Studies recommend that in surgical procedures where none of the above three indices justify a crossmatch, a blood group and save should suffice with serum saved for emergency crossmatching should the need arise $[4,7]$. Our results indicate that procedures such as laparoscopic or open fundoplication, laparoscopic or open cholecystectomy and total or hemithyroidectomy can be accommodated with only a group and screen. These results are in keeping with earlier work that showed a routine crossmatch was unnecessary for these and other procedures including mastectomy and duodenal ulcer surgery, excluding gastric resections.[1,3]. It has been further advocated that for procedures with significant utilization of blood the number of units to be crossmatched should be based on the ordering schedules of each hospital $[2,4,7]$. The maximum surgical blood ordering schedule (MSBOS) is a guide for the number of units of blood to be crossmatched for each elective surgical procedure and also determines which elective surgical procedures can be accommodated only with a group and screen $[2,4,5,8]$. An individual MSBOS needs to be established at each hospital by a hospital transfusion committee based on a retrospective analysis of actual blood usage of the hospital $[2,6,8,11]$. Studies have shown that developing a blood ordering schedule for each hospital improves the cost effectiveness of the hospital transfusion service[5,12].

This study demonstrates that crossmatching blood for selected surgical procedures exceeds requirement considerably - a practice we suspect is widespread in our healthcare system. These findings though not comprehensive, strengthen the case for the establishment of institution specific MSBOSs by hospital transfusion committees. The schedule requires regular monitoring and evaluation to verify compliance and institute updates. The potential benefits include improved availability of a vital resource and financial savings.

\section{References}

1. Smallwood JA. Use of blood in elective general surgery: an area of wasted resources. British Medical Journal 1983;286:868-870

2. Kozarzewska $M$ et al. The analysis of surgical blood order protocol. Anaesthesiology Intensive Therapy 2011;XLIII(2):61-63

3. Juma T, Baraka A, Abu-Lisan M, Asfar SK. Blood ordering habits for elective surgery:time for change. Journal of the Royal Society of Medicine 1990;83:368-370

4. Olawumi H, Bolaji B. Blood utilization in elective surgical procedures in Ilorin. The Tropical Journal of Health Sciences 2006;13:15-17

5. Richardson NGB, Donaldson DR, Bradley WN, O'Shaughnessy DF. Maximum surgical blood ordering schedule in a district general hospital saves money and resources. Ann R Coll Surg Engl 1998;80:262-265

6. Singh JK, Singh P. Routine pre operative cross match for elective colorectal resections: An appropriate use of resources? The Surgeon 2011:8-12

7. Ong YW, Vathsala A, Ng HW. An analysis of blood usage in elective surgery. Ann Acad Med Singapore 1984 Jul;13(3):531-7

8. Boral LI, Dannemiller FJ, Stanford W, Hill SS, Cornell TA. A guideline for anticipated blood usage during elective surgical procedures. Am J Clin Pathol 1979 Jun;71(6):680-4

9. Friedman BA, Oberman HA, Chadwick HR, Kington KI. The maximum blood order schedule and surgical blood use in the United States. Transfusion 1976;380-387.

10. Mead JH, Anthony CD, Sattler M. Hemotherapy in elective surgery : an incidental report, review of literature and alternatives for guideline approval. Am J Clin Path 1980;74:223-227.

11. Sarma DP. Use of blood in elective surgery. JAMA 1980 Apr 18;243(15):1536-8

12. Dodds AJ, Pun A, Isbister JP, Ting A, Klarkowski D, Concannon AJ, Biggs JC. A rational approach to cross matching blood for elective surgery. 